\title{
La educación del campesinado en el comercio internacional
}

\section{Educating the Peasantry in International Trade}

\author{
Angélica Vanessa Briñas Olaya ${ }^{10}$, Alison Jineth Barón Puentes"1", \\ Luisa María Murgueitio Trigos ${ }^{12}$, Emilio José Navarro Chávez $^{13}$
}

\section{Resumen}

En este documento se plasmó la problemática principal donde el campesinado juega el papel como protagonista, pues se describe que este grupo social carece de reconocimientos y garantías para tener una educación adecuada dentro del comercio internacional, siendo dependiente y sometido a decisiones de intereses por parte de terceros o por parte del Estado. Por eso se busca dar solución, o respuesta a ello, por medio de un proyecto educativo hacia el campesinado sobre comercio internacional, para que sean los suficientemente capaces de para ejercer su propio poder y capacidad de decisión dentro de la economía del país con el conocimiento idóneo, tanto a nivel nacional como a nivel internacional, siendo competentes con sus productos en el exterior, con el fin de dar el reconocimiento al campo como productor principal de la economía colombiana. Se investigó por medio de artículos y textos sobre los antecedentes de esta problemática para entender las razones de fondo de la problemática y, a partir de ello, se realizó una investigación para saber qué metodología podría ser la más idónea para llevar a cabo la educación del campesinado en cuanto a comercio internacional y hacer factible este proyecto. Por consiguiente, se garantiza

10 Estudiante, Fundación Universitaria del Área Andina, Programa de Negocios Internacionales, sede Bogotá. Correo electrónico: abrinas@estudiantes.areandina.edu.co

11 Estudiante, Fundación Universitaria del Área Andina, Programa de Negocios Internacionales, sede Bogotá. Correo electrónico: abaron14destudiantes.areandina.edu.co

12 Estudiante, Fundación Universitaria del Área Andina, Programa de Negocios Internacionales, sede Bogotá. Correo electrónico: Imurgueitiolestudiantes.areandina.edu.co

13 Estudiante, Fundación Universitaria del Área Andina, Programa de Negocios Internacionales, sede Bogotá. Correo electrónico: enavarro18Restudiantes.areandina.edu.co 
que la competencia de Colombia a nivel internacional representada por los productos del campo colombiano aporta al PIB del país a través una balanza comercial positiva.

Palabras clave: comercio internacional, desarrollo rural, educación rural, política rural.

\section{Abstract}

This paper reflects on the peasantry's major problem, as this social group lacks recognition and guarantees to receive an adequate education in international trade, being dependent on and subject to decisions by third parties or the State. As a solution, we propose an international trade educational project to provide peasants with sound knowledge and skills to exercise their power and decision-making within the country's economy and compete with their products abroad, thus recognizing the countryside as the leading producer of the Colombian economy. We reviewed articles and other documents on the background of this problem to understand its reasons fully. Then, we did some research to find out which method could be the most suitable to implement this project. Consequently, it is guaranteed that Colombia's international competitiveness represented by products from the countryside will positively contribute to its GDP.

Keyword: International trade, rural development, rural education, rural policy.

\section{Introducción}

Una de las problemáticas que más ha afectado al sector rural colombiano a lo largo de la historia, está relacionada con las deficientes políticas de desarrollo económico y social, asociadas a los medios de tecnificación del agro, al acceso a líneas blandas de créditos, a la disposición de tecnología e infraestructura, sumado a las precarias condiciones de co- mercialización y puesta de los productos en el mercado nacional e internacional. Lo cual, deja en evidencia la falta de información y bajas condiciones de acceso de este sector de la economía del país; igualmente, a las políticas de exportación y al conocimiento de las dinámicas globales de los mercados.

Esta situación, se convierte en un tema de relevancia para la investigación 
sobre los procesos de educación en temas de comercio y formulación de estrategias de mercado para el sector rural, de cara a la necesidad de diversificar la economía, ya que podría fortalecer los niveles de crecimiento social en el campo y el mejoramiento de la calidad de vida rural. En ese sentido, este proyecto se enfoca en presentar herramientas de gestión rural, con el fin de enfrentar de manera asertiva las nuevas condiciones de mercado, dinámicas de precios y optimización de recursos técnicos y humanos. Por tanto, es necesario atender de manera puntual las prácticas convencionales de comercialización formal e informal que se desarrollan en el sector para reducir las condiciones de inequidad y desigualdad social en el escenario de la comercialización de los productos del campo colombiano (Arias Gaviria, 2014). Cabe anotar que el sector rural presenta un alto nivel de incidencia en el renglón de la economía nacional, si se tiene en cuenta que gran parte de la industria nacional y el aprovisionamiento de alimentos en las grandes ciudades dependen de la producción rural del país. Sin embargo, al campesinado y agricultores no se les ha otorgado el lugar, respeto, apoyo y reconocimiento dentro de la economía nacional, ni como personas y sujetos derechos (Anderson et al., 2019).
A pesar de ser un motor del desarrollo y del dinamismo de las nuevas economías, el déficit educativo, como ya se mencionó en líneas anteriores, ha sido una constante en los niveles de cobertura, infraestructura y capital docente, necesarios para impulsar nuevas situaciones de desarrollo y competitividad en el campo. Los habitantes del sector rural se enfrentan a altos niveles de deserción escolar y cifras considerables de analfabetismo digital, como consecuencia del olvido por parte de las entidades territoriales, así como la falta de presencia del Estado y situaciones de violencia y deterioro de las condiciones sociales. Estas condiciones han limitado por décadas la participación estratégica del comercio rural frente a los mercados internacionales (Matijasevic Arcila, 2015).

Frente a este escenario poco promisorio para el sector rural de Colombia, algunos expertos señalan que es necesario proveer al campesinado de herramientas participación social, impulsar el desarrollo del componente educativo, apostar por la tecnificación y, ante todo, establecer puntos de conexión entre los productores primarios y los mercados, y reducir la intermediación; pero principalmente resignificar el papel del campesino, en los procesos de formulación de políticas públicas. Siguiendo a $\mathrm{Ru}$ - 
biano (2013), dotar de identidad y sentido de pertenencia y responsabilidad con el desarrollo del país al campesinado, permitirá la construcción de un nuevo modelo de desarrollo rural.

En consecuencia, se han de atender de manera oportuna "los diagnósticos sobre la situación educativa que coinciden con las zonas rurales donde se concentran los mayores índices de carencias" (Gajardo, 1988). En este sentido, resulta pertinente estructurar un plan educativo para el campesino que proporcione bases íntegras con respecto a los negocios y el comercio exterior, definir mecanismos y procesos de educación que permitan identificar temas pertinentes y asociados a las necesidad del agro (Cestille y Lima, 2009).

\section{Introducción}

Este ejercicio de revisión bibliográfica permitió evidenciar las principales situaciones de trasfondo que soportan el deterioro de la ruralidad en Colombia. En relación con esto, vale la pena resaltar que el campesinado ha sido afectado en diferentes aspectos que han minimizado su reconocimiento y valor dentro de la economía y la sociedad nacional, así lo señala Rubiano (2013). La información consultada establece que el ma- nejo inadecuado de temas como la salud, la educación, entre otros, han disminuido las condiciones de mejoramiento de la calidad de vida en el campo, ampliando la brecha entre lo urbano y lo rural (Lozano Flórez, 2012).

Analizar esta situación, ha permitido identificar situaciones humanas de desesperanza en los habitantes rurales, tensiones sociales y modelos naturales de supervivencia, según el contexto (Corrales y Forero, 2012). Indudablemente, estas condiciones de subdesarrollo que enfrenta el sector rural, denotan un interés superlativo por resignificar el modelo educativo en el campo, acerca la ciudad a las dinámicas rurales, proveer la formación profesional de un sentido de corresponsabilidad con el agro Colombiano y promover la articulación de la calidad educativa con las bases de la cultura rural (García, 2016).

Algunas investigaciones realizadas apuntan a la necesidad de potenciar el apoyo socioeconómico del campo, como estrategia para mejorar los niveles de producción agrícola y aportación de este sector a la economía nacional (Peña, 2014). Asimismo, se han desarrollado proyectos enfocados en analizar las problemáticas asociadas con el funcionamiento de las estructuras de comercia- 
lización de los productos agropecuarios del sector rural colombiano, en el marco de la globalización de los mercados (Ramírez Camacho, 2009).

\section{Contextualización}

Como se ha venido mencionado en líneas anteriores, la comercialización se constituye en uno de los temas más relevantes para impulsar la economía rural y el desarrollo de la economía nacional, más aún, cuando los estudiosos señalan la urgencia de cargar esto de institucionalidad, adoptando buenas prácticas y aplicando estándares de calidad a lo largo de la cadena productiva. En esta perspectiva, el comercio internacional como elemento coyuntural entre el comercio rural y el manejo de precios internacionales (exportaciones) impulsa el desarrollo social y la calidad de vida. Para ello, se ha de tener en cuenta el comportamiento de las tasas de cambio, dado que este contribuye a controlar el crecimiento y la diversificación de las exportaciones agropecuarias, sumado al comportamiento de la balanza comercial entre las exportaciones e importaciones y, de manera particular, el análisis de la participación de los productos agrícolas en esta dinámica.

Desde el punto de vista de la ruralidad y del desarrollo de la educación en este campo, la educación rural debe apuntar a la incorporación de las políticas públicas para el desarrollo del agro, promover la transformación social que contribuye a la formación integral de la persona, como lo establece la Ley 115 de 1994 (Tobón, 2019). De otro lado, la educación rural, debe estar acompañada de estrategias tecnológicas que le permitan al sector resolver las principales problemáticas del campo, así como la de atender las necesidades propias y del entorno inmediato. En síntesis, desarrollar competencias en el estudiante para el manejo de fuentes de información y formulación de propuestas de solución, asociados a las temáticas rurales (Gajardo, 1988).

Según Arias Gaviria (2017), se requiere poner en práctica las políticas educativas rurales, integradas al Plan Educativo Nacional. A partir de allí, impulsar un modelo educativo inclusivo y equitativo entre la educación de las urbes y la que se imparte en el contexto rural. Más aún, si se tienen en cuenta que a partir de la Constitución Política de 1991, Colombia propone una organización de Estado bajo un contexto de descentralización política, y a pesar de varias décadas bajo esta idea, Colombia aún no ha logrado establecer la igualdad y equidad de condiciones sociales y cul- 
turales que propone la descentralización política; si se logra esto podrían permitir el desarrollo de una sociedad, con una construcción de territorios competitivos, justos, democráticos y participativos, y ante todo, sostenibles del propio campo colombiano (Lozano Flórez, 2012).

La falla del sistema pedagógico en el país está en el planteamiento epistemológico que es dirigido al desarrollo de una sociedad liberal industrial, donde el maestro hace uso del poder de la autoridad y pierde ese factor humano para fomentar el desarrollo de una nación, y se rige por un proceso educativo exclusivo dirigido al privilegio (Lander, 2000; Peña, 2014).

Por los conocimientos reflexivos sobre la construcción de una sociedad, se puede saber que la autorreflexión e interacción social son fundamentales para el ejercicio de esta, en el que la contribución de sus ideas y acciones son influyentes para desempeñar un compromiso en lo ético y político. Esto genera el flujo de intercambio, construcción y transformación de la sociedad (Corrales y Forero, 2012).

Entre los planes de desarrollo de la nación, se destacan conceptos cruciales para el desarrollo y evolución de esta investigación, en este sentido, los pla- nes de Colombia después del 2000 se fundamenta en que hay un sistema judicial y de derechos humanos que incluye un plan de acción para priorizar el desarrollo alternativo, que promueva la función de la actividad agropecuaria y cualquier otra acción económica rentable para los campesinos y sus familias (Peña, 2014; Presidencia de la República de Colombia, 2000).

A partir de diferentes investigaciones sobre la raíz de la problemática campesina, gran parte del menosprecio hacia los campesinos se estandariza en la negación de derechos, negación como actores sociales y, por último, la desvalorización de sus formas de producción y sus productos finales (Matijasevic Arcila, 2015).

El escenario al que se enfrentan los campesinos se explica principalmente por razones políticas y económicas. Entre las primeras, se encuentra un discurso político del desarrollo agrario basado en la competitividad, la producción agroindustrial y el privilegio a la actividad exportadora de productos con ventajas comparativas (Ramírez Camacho, 2009).

Según (Beltrán et al., 2016), se señala dos ámbitos en los cuales son reconocidos el campesinado: por un lado, los campesinos rurales son catalogados como uno obstáculo para la transforma- 
ción social y, por otro lado, se les reconoce como guardianes del ambiente y vitales para el futuro agrario del país. Este primer ámbito señalado anteriormente, permite ver el concepto mayormente generalizado de lo que es el campesinado, mientras la otra nos da esta perspectiva a la que se busca llegar, mostrando al campesino como conocedor y productor de las tierras colombianas, como el principal potenciador y proveedor de la manutención de la economía de un país, tanto en el presente como en el futuro. Es ahí cuando la tierra cobra valor para la economía de un país y debe ser valorada y potencializada. Un campo conocedor de los beneficios de sus tierras para su propio país es el perfecto ideal para encaminar la educación hacia el comercio exterior y competencia independientemente, lo que genera que la balanza comercial del país se incline hacia el lado positivo.

Por otro lado, Mora et al. (2011) analiza lo que ha significado para el país, en general, y para el campesinado, en particular, el haber identificado el crecimiento con el desarrollo, sin preocuparse por los dilemas distributivos, por la inclusión y el reconocimiento político, por la protección del patrimonio material e inmaterial de la sociedad rural y por la sostenibilidad ambiental. Según Gaviria (2017), la educación rural se suele considerar como la forma de consumo de tecnología, como técnica de ingreso a la sociedad sin estudio y la manera de mostrar una especie de cercanía al mundo modernizado, en el cual, la educación rural transmite un currículo general, de carácter nacional, que deja por fuera el saber propio de la población campesina (Gajardo, 2010). La deserción y la repetición, la falta de equipos y edificios adecuados, la poca funcionalidad de los modelos educativos y la carencia de docentes formados son claramente los problemas más específicos de la población escolar.

Corrales y Forero (2012) explican que esto impacta de manera clara en el sector agrario, pues será prioritario que los productores puedan adquirir los conocimientos suficientes para usar tecnología que le permita realizar las tareas de una forma mucho más eficiente teniendo en cuenta los resultados y el tiempo ahorrado; todo esto para lograr una mejor posición en el mercado.

\section{Función principal de la investigación}

Anderson et al. (2019) señala que, para llegar a realizar este procedimiento es necesario reconocer el compromiso de la educación crítica campesina, ya que es la clave para el crecimiento del país y el futuro en la producción de alimentos, 
agricultura y transformación social. Con total certeza, el aprendizaje impulsa la visión que transformaría a la población campesina con respecto a sus movimientos sociales y sus ideales educativos.

Todo esto con el fin de poder cumplir a su vez los objetivos que tiene la Presidencia de Colombia desde el 2000 para el desarrollo del país. Para llegar a estos objetivos, el Ministerio de Educación sugirió la propuesta, en un primer momento, sobre los principales aspectos a tratar en cuanto al problema educativo, los cuales deben abarcar desde la cobertura y la permanencia. Sin embargo, estudios previos interpretan factores que claramente son mucho más relevantes y que pueden generar resultados mucho más convincentes como lo son la calidad, el estudio de los modelos pedagógicos flexibles, la educación rural en secundaria y media, una visión cultural y los imaginarios sociales (Valencia 2015; Rodríguez y Rodríguez, 2020).

Dado lo anterior, se puede destacar que la Educación profesional del campo está, y como la afirman Cestille y Lima (2010), concretándose y que esto tiene un propósito, y abarca mucho más allá que cualquier dimensión pedagógica y metodológica referenciales del modelo educativo. Ya que toma como figura el contexto que envuelve a la cultura del campo y la profundización de su humanidad, pues que velan por sus derechos humanos para abarcarlos y lograr con mayor eficacia integrarlos a la sociedad como seres sociales de vital importancia.

A partir de la situación planteada por Álvarez (2018), en la cual nos muestra la importancia del género y del desarrollo de identidades del campesino rechazado, el cual es afectado por las desplazamientos forzados, obligándolos a enfrentar a ciudades capitales, donde debido a su deficiencia educativa y de competencias dentro de la economía nacional, se ven obligados a vivir en barrios marginales con condiciones de deplorables de vida, que suman al índice de pobreza colombiana.

De lo anterior citado, es importante la implementación del proyecto, puesto que influirá de forma positiva en la vida del campesinado, pues podría dar cambio a esta realidad, ya el campesino estaría apto para laborar independientemente en el comercio internacional con sus productos derivados $100 \%$ del campo colombiano.

\section{Resultados}

Las investigaciones realizadas se organizaron principalmente para reconocer que tanto conocimiento había en Bogotá, 
la capital de Colombia, sobre la situación actual educacional campesina y que tan cercanos se encuentran a esta población en cuanto a sus raíces familiares. Es importante poder conocer qué tanto impacto tiene este tipo de problemáticas en ciudades metrópolis, donde no se tiene tanto conocimiento de qué sucede alrededor en la población rural. La muestra encuestada obedeció a 25 participantes que, en un gran porcentaje, se ubican los estratos 3 y 4 , y en su mayoría tienen edades superiores a $\operatorname{los} 30$ años.

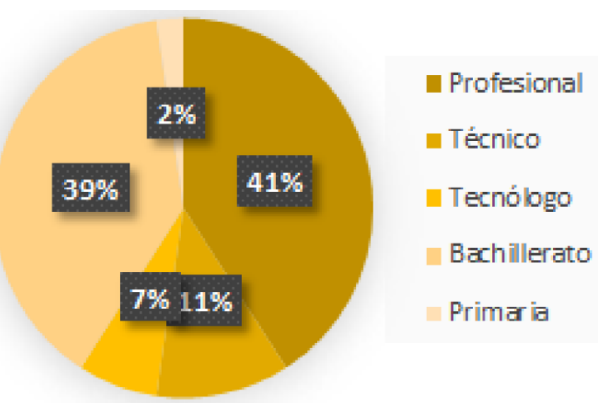

Figura 1. Nivel educativo.

Fuente: elaboración propia.

El nivel educacional con mayor porcentaje es el profesional con un 40,9\% (figura 1), siguiendo con el 38,6\% de los encuestados quienes tienen título de bachiller. Por otro lado, se obtuvo que $11,4 \%$ de los encuestados tienen el título técnico. Mientras que el 6,8\% son tecnólogos y, finalmente, el 2,3\% solamente tiene estudios hasta la primaria.

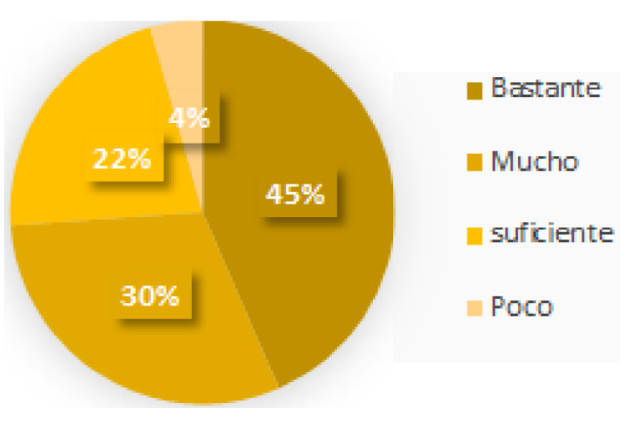

Figura 2. Campesino en la sociedad.

Fuente: elaboración propia.

El 61,4\% de los encuestados opina que el campesino es bastante importante en la sociedad (figura 2), mientras, por otro lado, el $36,4 \%$ diferencia que el campesino es mucho más que importante en la sociedad. Concluyendo solo el 2,4\% considera que el campesino es más o menos importante en la sociedad.

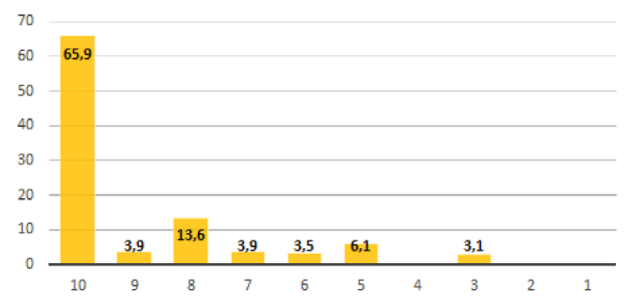

Figura 3. Educación del campesino. Fuente: elaboración propia.

El 65,9\% de los encuestados indicó, por una diferencia mucho más alta, el número 10 de la escala, sosteniendo que es necesario que los campesinos reciban educación para desempeñar roles inde- 
pendientes en el comercio internacional (figura 3); seguido de $13,6 \%$ con una puntuación de $8 ; 6,1 \%$ con un valor de 5; 3,9\% marcados de la misma manera para los valores 7 y $9 ; 3,5 \%$ en el valor 6 y $3,1 \%$ para el valor marcado como 3.

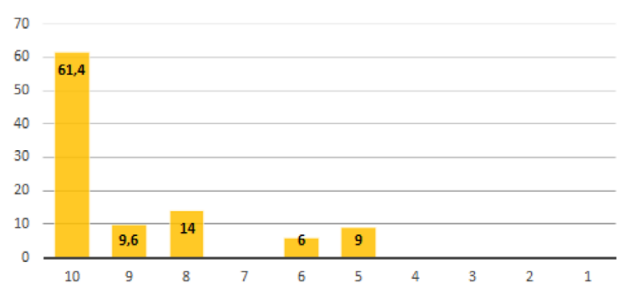

Figura 4. Educación de calidad. Fuente: elaboración propia.

En la figura 4, se puede denotar que los resultados favorecen a que la mayoría de los participantes opinan que la economía si mejoraría si los campesinos contarán con una mejor educación. Con respecto a lo anterior, se puede destacar que el porcentaje más alto de la escala pertenece al valor correspondiente al número 10 con el $61,4 \%$, el valor 8 representa el $14 \%$; valor 9 es para el 9,6\%; el valor 5 constituye el $9 \%$, y finalmente el valor de 6 para el $6 \%$ de los encuestados.

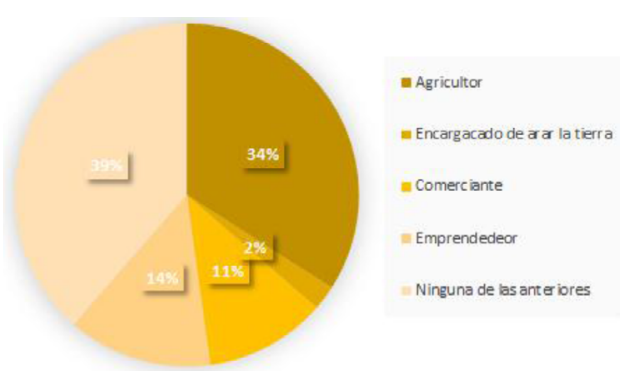

Figura 5. Familia campesina.

Fuente: elaboración propia.

El 38,6 \% de los encuestados responde que no tiene familia campesina siendo el mayor porcentaje en esta pregunta (figura 5), mientras que el 34,1 $\%$ tiene familiares campesinos que son agricultores. Por otro lado, el 13,6 \% tienen familiares campesinos que realizan actividades, el 11,4 \% tiene familiares campesinos que son comerciantes, $y$, finalmente, el 2,3\% de los encuestados tienen familiares campesinos que son encargados de arar la tierra.

\section{Discusión y conclusiones}

La realización de la encuesta permitió conocer aspectos muy importantes con respecto a la ciudad de Bogotá y que conocimientos se tiene sobre la problemática del ámbito educacional campesino.

Fue importante saber los conocimientos generales y la opinión de personas no 
ligadas a la vida del campesino, esto impacta los resultados, ya que la igualdad de acceso a los derechos fundamentales de cualquier ciudadano comienza por la educación pública y de calidad, porque garantiza la emancipación como seres sociales (Cestille y Lima, 2009).

En primera instancia, se encontró que la gran mayoría de personas encuestadas se encontraban entre los estrato 2 y 3 , dada la representación de conocimiento general en los diferentes estratos económicos y su perspectiva. Es aquí, donde sabemos con certeza que la gran mayoría de los encuestados cuenta con un nivel educativo encima del bachillerato, por ende, con capacidad de un criterio propio (Anderson et al., 2019).

Cabe destacar que, las preguntas realizadas se hicieron de manera clara y específica no solo para la obtención de resultados de manera sencilla, sino que se procuró que, por medio de la encuesta, los encuestados pudieran conocer cuál es el foco de la atención del proyecto y porqué es importante el debate del pensamiento crítico como sociedad.

Con base en la gran mayoría de respuestas de la encuesta, se pudo constatar que los encuestados están de acuerdo con que el campesinado no ha sido favorecido en cuanto a tener una educación de calidad, y refieren que este es un factor que golpea directamente a la economía (Perfetti, 2009).

La independencia de comercialización en la producción campesina es un resultado que se refleja en la encuesta, incluso sorprendió con un alto porcentaje de los encuestados, debido a que los campesinos son los que producen un alto porcentaje de alimentos consumidos por el país, aportando a la económica, ya que mantiene el sistema agroalimentario de la nación relativamente autosuficiente (Corrales y Forero, 2012).

Según los resultados obtenidos en nuestra encuesta, se puede concluir que los métodos para lograr un beneficio para esta población tiene una relación directa con los de otras investigaciones afines, ratificando el valor de la educación y la falta de la misma dentro de la población rural, no solo en Colombia sino en América Latina (Perfetti, 2009).

La importancia de la pedagogía dentro del proyecto educativo se evidencia en la necesidad de que los maestros tener un tipo de educación extra y específica que se adapte al contexto cultural del área rural en donde se vayan a desempeñar, partiendo de la paz como base fundamental (Rosales, 2009). 


\section{Referencias}

Álvarez, N. H. (2018). El género: una categoría necesaria para comprender la experiencia del desplazamiento forzado en Colombia. Inclusión y Desarrollo, 5(1), 49-55. https://doi.org/10.26620/uniminuto.inclusion.5.1.2018.49-59

Anderson, C. R., Binimelis, R., Pimbert, M. P., \& Rivera-Ferre, M. G. (2019). Introduction to the symposium on critical adult education in food movements: learning for transformation in and beyond food movements-the why, where, how and the what next? Agriculture \& Human Values, (36), 521-529. https://doi.org/10.1007/ s10460-019-09941-2

Arias Gaviria, J. (2014). Educación rural y saberes campesinos en Tierradentro, Cauca: estudio del proceso organizativo de la Asociación Campesina de Inzá Tierradentro (ACIT). 2004 a 2012 [tesis de grado, Universidad Nacional de Colombia, Bogotá]. https://repositorio.unal.edu.co/handle/ unal/52109

Arias Gaviria, J. (2017). Problemas y retos de la educación rural colombiana. Revista Educación y Ciudad, (33), 53-62. https://doi. org/10.36737/01230425.v0.n33.2017.1647

Beltrán, A. M., Cadavid, M. J., Castillo, O. L., Espinosa, N., Ferro, J. G., Herrera, J., Herrera, M., Jaramillo, O. E., Méndez, Y. A., Osorio, F. E., Sierra, A. M., Tobón, G. J., Uribe, G. y Vargas, N. (2016). Dime que paz quieres y te diré que campo cosechas. Bogotá: Pontificia Universidad Javeriana. https://problemasrurales.files.wordpress. com/2008/12/dime-que-paz-quieres-y-tedire-que-campo-cosechas-20161.pdf
Gajardo, M. (1988). Enseñanza básica en las zonas rurales: experiencias innovadoras. Santiago de Chile: unesco, OREALC. https://unesdoc.unesco.org/ark:/48223/ pf0000084246

Lozano Flórez, D. (2012). Contribuciones de la educación rural en Colombia a la construcción social de pequeños municipios y al desarrollo rural. Revista de la Universidad de La Salle, (57), 117-136. https://ciencia.lasalle.edu.co/ruls/vol2012/ iss $57 / 7 /$

Matijasevic Arcila, M. T. (2015). Experiencias de reconocimiento y menosprecio en campesinas y campesinos de Caldas [tesis doctoral, Universidad de Manizales, CINDE, Manizales]. http:// biblioteca.clacso.edu.ar/Colombia/alianza-cinde-umz/20160121124205/MariaTMatijasevic.pdf

Mora, A., Tolosa, Á., Pinilla, C., García, C., Lombo, E., Naranjo, S. y Clavijo, P. (2011). El campesinado. Reconocimiento para construir país. Bogotá: Programa de las Naciones Unidas para el Desarrollo (PNUD). https://www.undp. org/content/dam/colombia/docs/DesarrolloHumano/undp-co-cuadernocampesinado-2012.pdf

Peña, C. M. (2014). Conocimiento escolar y saberes campesinos. Encuentros y desencuentros en la escuela rural. Praxis Pedagógica, 14(15), 103-123. https://doi.org/10.26620/ uniminuto.praxis.14.15.2014.103-123

Presidencia de la República de Colombia. (2000). Plan Colombia: plan para la paz, la prosperidad y el fortalecimiento del Estado. Bogotá: Imprenta Nacional de Colombia. 
Ramírez Camacho, D. (2009). Estrategias de supervivencia campesina: alternativas organizativas para mercados especializados. El caso del municipio El Colegio, Cundinamarca [tesis de grado, Universidad del Rosario, Bogotá]. https://repository.urosario.edu.co/bitstream/handle/10336/1784/ RamirezCamacho-Diana-2009.pdf?sequence $=1 \&$ isAllowed $=\mathrm{y}$

Rodríguez-Rodríguez, M. P. (2020). Aportes de la práctica pedagógica en las comunidades rurales del municipio de Restrepo, Departamento del Meta (Colombia). Orinoquia, 24(1), 77-85. https://doi. org/10.22579/20112629.593 